Forum 2020 · 35:368-372

https://doi.org/10.1007/s12312-020-00834-8

Online publiziert: 4. September 2020

(c) Springer Medizin Verlag $\mathrm{GmbH}$, ein Teil von Springer Nature 2020

Die Zulassung bzw. Indikationsausweitung für alle onkologischen Arzneimittel erfolgt heute in der Europäischen Union (EU) auf Grundlage der Verordnung der Europäischen Gemeinschaft (EG) Nr. 726/2004 in einem zentralisierten Verfahren der European Medicines Agency (EMA). Sie erfordert klinische Studien der Phase II bzw. III, an deren Durchführung von der EMA detaillierte regulatorische Anforderungen gestellt werden - zuletzt in einem Konzeptpapier, das die derzeit gültige Richtlinie zur „Evaluation of anticancer medicinal products in man" (EMA/CHMP/205/95 Rev 5) ersetzen soll [1].

\section{》) Entscheidender Endpunkt für klinische Studien in der Onkologie sind günstige Effekte auf das OS}

In diesem Konzeptpapier wird für konfirmatorische Studien der Phase III gefordert, dass ein positives Nutzen-RisikoProfil („,benefit-risk ratio“) einschließlich notwendiger supportiver Maßnahmen nachgewiesen werden soll sowohl für ein neues Arzneimittel als auch für ein bereits zugelassenes onkologisches Arzneimittel mit neuem Anwendungsgebiet, und zwar in einer gut charakterisierten

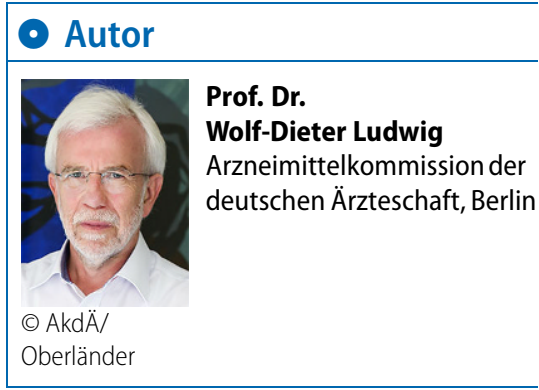

Wolf-Dieter Ludwig

Arzneimittelkommission der deutschen Ärzteschaft, Berlin, Deutschland

\title{
Klinische Endpunkte in Studien
} Was ist relevant für HTA und Patienten?

Zielpopulation, die für den klinischen Alltag relevant ist. Im Abschnitt zur Patientenpopulation werden dabei folgende Parameter ausführlich beschrieben [1]:

- geeignete Vergleichstherapie;

- Vorgehen bei der Randomisierung;

- Verblindung der Phase-III-Studie;

- Endpunkte, die in konfirmatorischen Studien den klinischen Nutzen belegen sollen.

Als akzeptable primäre Endpunkte werden genannt [1]:

- Heilungsrate,

- Gesamtüberleben („overall survival“ [OS]),

- progressionsfreies bzw. erkrankungsfreies Überleben („progression-free/ disease-free survival“ [PFS bzw. DFS]).

Entscheidender Endpunkt für klinische Studien in der Onkologie sind aus klinischer, aber auch aus methodischer Sicht plausibel nachgewiesene günstige Effekte auf das OS. Daneben kann eine Verlängerung des PFS bzw. des DFS jedoch ebenfalls einen Nutzen für den Patienten anzeigen. Die Auswahl des primären Endpunkts sollte sich auch an der relativen Toxizität der neuen (experimentellen) Therapie orientieren und dabei das zu erwartende Überleben nach Progression der Tumorerkrankung, verfügbare Zweitlinientherapien und die Prävalenz der Tumorerkrankung berücksichtigen [1]. Wenn in konfirmatorischen Studien das PFS/DFS als primärer Endpunkt ausgewählt wird, sollten das OS als sekundärer Endpunkt und bei Auswahl des OS als primärer Endpunkt entsprechend das PFS/DFS als sekundärer Endpunkt untersucht werden.

Darüber hinaus wird in der überarbeiteten, aber noch nicht endgültig verab- schiedeten Richtlinie gefordert, dass unabhängig von der Auswahl des primären Endpunkts auch Angaben zur Stabilisierung der Tumorerkrankung (z. B. über 3 oder 6 Monate) gemacht werden, ebenso wie in der palliativen Situation zur gesundheitsbezogenen Lebensqualität bzw. zu den aus Sicht des Patienten positiven oder negativen Auswirkungen der medikamentösen Behandlung (,patient-reported outcomes" [PRO]; [1]). Außerdem werden detaillierte Vorgaben gemacht hinsichtlich des zu erbringenden Nutzen-Risiko-Verhältnisses des neuen Arzneimittels (Überlegenheit vs. Nichtunterlegenheit), jeweils in Abhängigkeit von der Zielsetzung der Therapie (kurativ, langfristige Krankheitskontrolle, palliativ) und ihrer zu erwartenden Toxizität [1].

Zulassungsstudien - in der Onkologie, aber auch in den meisten anderen Anwendungsgebieten von Arzneimitteln - werden heute größtenteils von pharmazeutischen Unternehmern (pU) gesponsert, konzipiert und ausgewertet [2]. Sie verfolgen das Ziel, möglichst schnell die Marktzulassung für das neue Arzneimittel bzw. ein neues Anwendungsgebiet zu erhalten. Wissenschaftliche Untersuchungen in Europa und Nordamerika haben in den letzten Jahren auf zahlreiche Defizite in den für die Zulassung onkologischer Arzneimittel relevanten („pivotal“) klinischen Studien hingewiesen [2-5]. Sie betreffen v. a. [2-7]:

- Studiendesign,

- meist (zu) restriktiv definierte Einund Ausschlusskriterien für Patienten,

- Verwendung von Surrogat- anstelle von harten Endpunkten,

- Veränderungen im Studiendesign nach Beginn der Patientenrekrutierung (insbesondere bei beschleu- 
Hier steht eine Anzeige.

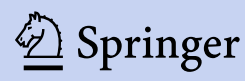


nigten Zulassungen und einarmigen klinischen Studien),

- vorzeitiger Abbruch der Studien mit Wechsel der Patienten aus der Kontrollgruppe in den experimentellen Arm,

- zu kurze Zeiträume der Nachbeobachtung,

- vermehrtes Auftreten von Nebenwirkungen nach Änderungen der Anwendungsgebiete ohne Prüfung in einer randomisierten kontrollierten Studie.

Verantwortlich hierfür sind insbesondere die in den letzten 25 Jahren - zunächst in den USA und seit 1995 auch in Europa - eingeführten beschleunigten Zulassungsverfahren [2]. Inzwischen wird in den USA und in Europa die Mehrzahl der onkologischen Arzneimittel zur Behandlung von schwerwiegenden Erkrankungen in beschleunigten Zulassungsverfahren beurteilt, die z. B. in den USA mit einer verkürzten Begutachtung ( 6 anstelle von 10 Monaten bei normalen Verfahren) durch die Food and Drug Administration (FDA), intensiver Beratung durch die FDA bzw. EMA sowie Akzeptanz von Surrogatendpunkten als Beleg für den Nutzen des jeweiligen Arzneimittels verbunden sind [2-5]. In der EG wurde als beschleunigtes $\mathrm{Zu}$ lassungsverfahren bereits im Jahr 1995 die Zulassung unter außergewöhnlichen Umständen und später die beschleunigte Beurteilung, bedingte Zulassung („,conditional marketing authorisation“ [CMA]) sowie zuletzt im April 2016 das sog. PRIME(„priority medicines“)Verfahren eingeführt, v.a. für Arzneimittel für neuartige Therapien (z.B. Gentherapien; [2]).

\section{I) Neben der beschleunigten Beurteilung erfolgt heute am häufigsten die bedingte Zulassung}

Neben der beschleunigten Beurteilung wird heute am häufigsten die bedingte Zulassung anhand noch unvollständiger klinischer Daten erteilt für Arzneimittel, die zur Behandlung, Vorbeugung oder ärztlichen Diagnose von zu schwerer Invalidität führenden oder lebensbedrohlichen Krankheiten bestimmt sind, für Orphan-Arzneimittel oder für Arzneimittel, die in Krisensituationen gegen eine Bedrohung der öffentlichen Gesundheit eingesetzt werden sollen (vgl. CMA für Remdesivir bei COVID-19 [„, coronavirus disease 2019“] [2, 3]). Die Erteilung der bedingten Zulassung ist verknüpft mit der Anforderung, dass weitere umfangreiche Daten zur Wirksamkeit und Sicherheit nach der Zulassung bei der EMA eingereicht werden und ein sog. ungedeckter medizinischer Bedarf (,,unmet medical need") in dem jeweils zugelassenen Anwendungsgebiet besteht [2].

Bedingte Zulassungen sind nur ein Jahr gültig, werden jährlich überprüft und nach Erfüllung der von der EMA mit der Zulassung erteilten Auflagen (z.B. Vorlage von weiteren Ergebnissen $\mathrm{zu}$ Nutzen und Sicherheit aus laufenden oder neuen klinischen Studien) in eine reguläre Zulassung umgewandelt. Nach Auswertungen der EMA sind von 2006 bis 2018 insgesamt 38 Arzneimittel in Europa bedingt zugelassen worden, von denen nach Erfüllung der zuvor genannten Auflagen 19 in eine reguläre Zulassung umgewandelt werden konnten [2]. Seit Inkrafttreten der frühen Nutzenbewertung in Deutschland im Jahr 2011 wurde bis Ende 2018 bei insgesamt 15 dieser Arzneimittel zur Behandlung onkologischer Erkrankungen (solide Tumoren, hämatologische Neoplasien) der Zusatznutzen bewertet. Er war bei 12 der 15 Arzneimittel nicht belegt, nicht quantifizierbar oder gering $[2,8]$.

Die häufig zum Zeitpunkt der Zulassung unzureichende Evidenz für eine bessere Wirksamkeit oder Sicherheit der neu zugelassenen onkologischen Wirkstoffe im Vergleich mit der zweckmäßigen Vergleichstherapie bedeutet ebenso wie bei anderen beschleunigten Zulassungsverfahren, dass Onkologen bei der Verordnung den klinischen Nutzen, aber auch die Risiken neuer Arzneimittel nicht eindeutig beurteilen und dementsprechend die Patienten hierüber auch nicht adäquat informieren können [2, 3, 9].

Der Status quo der zum Zeitpunkt der Zulassung vorliegenden Evidenz für
Wirksamkeit bzw. Risiken bei neuen onkologischen Arzneimitteln wurde in den USA und in Europa in diversen Übersichtsarbeiten bzw. systematischen Reviews analysiert, insbesondere hinsichtlich der Konsequenzen, die aus der zunehmenden Verwendung von Surrogatendpunkten (z. B. Ansprechrate, PFS, DFS) in beschleunigten Zulassungsverfahren resultieren [2-6]. Diese Analysen ergaben übereinstimmend, dass die Verwendung von Surrogatendpunkten die Durchführung klinischer Studien zu Onkologika beschleunigen kann, nur selten jedoch während der Nachbeobachtung eine Verlängerung des OS oder zumindest eine Verbesserung der Lebensqualität als patientenrelevanter Nutzen überzeugend gezeigt werden kann und zudem weitere klinische Studien nach der Zulassung zum Nutzen der neuen Arzneimittel nur selten durchgeführt werden [3-6]. Es ist deshalb auch nicht überraschend, dass in den insgesamt 193 Beschlüssen des Gemeinsamen Bundesausschusses (G-BA) zu den im Zeitraum zwischen Anfang 2011 und Mai 2020 abgeschlossenen Verfahren der frühen Nutzenbewertung zu Onkologika, darunter auch viele Orphan-Arzneimittel, der Zusatznutzen gegenüber der zweckmäßigen Vergleichstherapie in mehr als der Hälfte der Beschlüsse als nicht belegt, nicht quantifizierbar oder (sehr selten) als geringer bewertet wurde [8].

\section{Ausblick}

Die aus Sicht der Wissenschaftler eher laxe Zulassungspraxis in der Onkologie, insbesondere im Rahmen beschleunigter Zulassungsverfahren und bei der Zulassung von Orphan-Arzneimitteln, häufig verbunden mit systematischen Fehlern in Design und Auswertung randomisierter kontrollierter Studien in der Onkologie [3] wurde in den letzten Jahren häufig kritisiert, und es wurde ausdrücklich hingewiesen auf mögliche Konsequenzen dieser meist auf unzureichender Evidenz basierenden Zulassungen [2, 3, 6, 9]:

- Unsicherheit und möglicherweise Schaden für individuelle Patienten;

- Verschwendung wichtiger Ressourcen in solidarisch finanzierten 
Gesundheitssystemen infolge Verordnung neuer (meist sehr teurer) Onkologika;

- Beeinträchtigung der Verabreichung notwendiger, erschwinglicher medikamentöser Behandlungen.

Dieser Kritik wurde aus Sicht der EMA widersprochen, da die Forderung, nur Arzneimittel mit einem gesicherten $\mathrm{Zu}$ satznutzen (z.B. in der Onkologie) zuzulassen, den derzeit geltenden Anforderungen an die Zulassung (der Nutzen eines neuen Arzneimittels überwiegt dessen Risiken) nicht entsprechen und zudem die wissenschaftlich gerechtfertigte Flexibilität hinsichtlich Entwicklung und Zulassung missachten würde [12].

Da die zuvor genannten Probleme in den Zulassungsstudien zu Onkologika durch die revidierte Richtlinie zur „Evaluation of anticancer medicinal products in man" der EMA [1] sicher nicht bald behoben werden können, ist eine verstärkte und auch politisch angestrebte Zusammenarbeit des G-BA bzw. des Instituts für Qualität und Wirtschaftlichkeit im Gesundheitswesen (IQWiG) mit dem Europäischen Netzwerk der HTA-Institutionen (EUnetHTA) und der EMA (z. B. im Rahmen der „Early Dialogues“ bzw. des „Scientific Advice“) sehr wichtig, auch um sich aktiv an der Planung des Designs und der relevanten Endpunkte in den für die Zulassung entscheidenden Studien der Phase III in der Onkologie zu beteiligen. Aus Sicht der Arzneimittelkommission der deutschen Ärzteschaft (Akd ̈̈) sollte in diesen klinischen Studien vor der Zulassung von Onkologika auch künftig die Untersuchung der gesundheitsbezogenen Lebensqualität bzw. der PRO verstärkt erfolgen $[10,11]$.

Darüber hinaus ist es in der Onkologie angesichts der häufig unvollständigen oder sogar fehlenden Evidenz zum Nutzen neuer Arzneimittel zum Zeitpunkt des Markteintritts von zentraler Bedeutung, dass nach Zulassung verpflichtend weitere klinische Untersuchungen zur Generierung versorgungsnaher Daten durchgeführt bzw. Daten aus qualitativ hochwertigen Registern vermehrt genutzt werden. Hierfür sind die im Sozialgesetzbuch (SGB) V (\$35a) im Rahmen des Gesetzes für mehr Sicherheit in der Arzneimittelversorgung (GSAV) neu vorgesehenen anwendungsbegleitenden (versorgungsnahen) Datenerhebungen (vgl. Beitrag von Thomas Kaiser und Beate Wieseler) sicher relevant, v.a. bei Befristungen im Rahmen der frühen Nutzenbewertung, ebenso wie eine konsequente Anordnung von „post-authorisation safety/efficacy studies" durch die EMA.

Angesichts der zunehmenden Bedeutung von genomischen Daten als Biomarker und Motor der sog. Präzisionsmedizin in der Onkologie sowie des zunehmenden Einsatzes adaptiver Studiendesigns (z. B. Basket-, Umbrellaund Platform-Studien) für die Entwicklung und Zulassung von Onkologika in klinischen Studien der Phasen I bis III ist es zunehmend wichtig, auf die Probleme bei der Generierung überzeugender Evidenz durch neue, auf molekularen Befunden basierende Ergebnisse hinzuweisen und die Kausalität von Zusammenhängen zwischen den Ergebnissen dieser Studien und molekularen bzw. klinischen Befunden regelmäßig $\mathrm{zu}$ überprüfen [13-16]. In diesen interventionellen Studien werden häufig grundsätzliche Fragen hinsichtlich der Auswirkungen dieser Arzneimittel auf wichtige Endpunkte wie Überleben und Lebensqualität nicht untersucht und zudem die für neuartige Therapiestrategien in Frage kommenden Patientenpopulationen, die Behandlungsdauer sowie die Sequenz bzw. Kombination von Wirkstoffen ungenügend charakterisiert [14].

Die z. T. sehr unterschiedlichen Sichtweisen von Pharmakoepidemiologen, nicht auf Profit ausgerichteten onkologischen Expertenkommissionen sowie an klinischen Studien aktiv beteiligten Onkologen hinsichtlich des geeigneten Designs und der Endpunkte klinischer Studien zur Zulassung neuer Arzneimittel in der Onkologie verdeutlicht eindrucksvoll ein kürzlich in Nature Reviews Clinical Oncology publizierter Artikel [16].

\section{Korrespondenzadresse}

Prof. Dr. Wolf-Dieter Ludwig

Arzneimittelkommission der deutschen

Ärzteschaft

Herbert-Lewin-Platz 1, 10623 Berlin,

Deutschland

wolf-dieter.ludwig@akdae.de

\section{Einhaltung ethischer Richtlinien}

Interessenkonflikt. W.-D. Ludwig gibt an, dass kein Interessenkonflikt besteht.

Für diesen Beitrag wurden von den Autoren keine Studien an Menschen oder Tieren durchgeführt. Für die aufgeführten Studien gelten die jeweils dort angegebenen ethischen Richtlinien.

\section{Literatur}

1. European Medicines Agency (2019) Concept paper on the revision of the guideline on the evaluation of anticancer medicinal products in man. https:// www.ema.europa.eu/en/evaluation-anticancermedicinal-products-man. Zugegriffen: 20. Juli 2020

2. Ludwig W-D (2019) Zulassungsverfahren für neue Arzneimittel in Europa. In: Schwabe U, Paffrath D, Ludwig W-D, Klauber J (Hrsg) ArzneiverordnungsReport 2019.Springer, Berlin

3. Naci H, Davis C, Savovic J, Higgins JP, Steme JAC, Gyawali B, Romo-Sandoval X, Handley N, Booth CM (2019) Design characteristics, risk of bias, and reporting of randomised controlled trials supporting approvals of cancer drugs by European Medicines Agency, 2014-16: cross sectional analysis. BMJ 366:15221

4. Gyawali B, Hey SP, Kesselheim AS (2019) Assessment of the clinical benefit of cancer drugs receiving accelerated approval. JAMA Intern Med 179(7):906-913

5. Chen EY, Haslam A, PrasadV (2020) FDA acceptance and surrogate endpoints for cancer drug approval: 1992-2019. JAMA Intern Med 180:912-914

6. Schuster Bruce C, Brhlikova P, Heath J, McGettigan P (2019) The use of validated and nonvalidated surrogate endpoints in two European Medicines Agency expedited approval pathways: a crosssectional study of products anthorised 2011-2018. PLoS Med 16(9):e1002873. https://doi.org/10. 1371/journal.pmed.1002873

7. Shepshelovich D, Tibau A, Goldvaser H, Molto C, Ocana A, Seruga B, Amir E (2018) Postmarketing modifications of drug labels for cancer drugs approved by the US Food and Drug Administration between 2006 and 2017 with and without supporting randomized controlled trials. J Clin Oncol 36(18):1798-1805

8. Gemeinsamer Bundesausschuss Nutzenbewertung von Arzneimitteln gemäß $\S$ 35a SGB V. https://www.g-ba.de/themen/ arzneimittel/arzneimittel-richtlinie-anlagen/ nutzenbewertung-35a/.Zugegriffen: 20 . Juli 2020

9. Wieseler B, McGauran N, Kaiser T (2019) New drugs: where did we go wrong and what can we do better? BMJ366:14340.https://doi.org/10.1136/bmj.I1430

10. Hwang TJ, Gyawali B (2019) Association between progression-free survival and patients' quality 


\section{Veranstaltungstipp}

of life in cancer clinical trails. Int J Cancer 144:1746-1751

11. Gnanasakthy A, Barrett A, Evans E, D'Alessio $D$, Romano C (2019) A review of patient-reported outcomes labeling oncology drugs approved by the FDA and the EMA (2012-2016). Value Health 22:203-209

12. Eichler HG, Enzmann H, Rasi G (2019) Added therapeutic benefit and drug licensing. Nat Rev Drug Discov 18(9):651-652

13. Park JJH, Siden E, Zoratti MJ, Dron L, Harari O, Singer J, Lester RT, Thorlund K, Mills EJ (2019) Systematic review of basket trials, umbrella trials, and platform trials: a landscape analysis of master protocols. Trials 20:572-583

14. Lacombe D, Bogaerts J, Tombai B, Maignen F, Osipienko L, Sullivan R, Golfinopoulos V (2019) Late translational research: putting forward a new model for developing new anti-cancer treatments that addresses the needs of patients and society. MolOncol 13:558-566

15. Ludwig W-D (2019) Kritische Analyse der Präzisionsmedizin am Beispiel der Onkologie. IMABE 26(3):119-129

16. Kurzrock R, Kantarjian HM, Kesselheim AS, Sigal EV (2020) New drug approvals in oncology. Nat Rev Clin Oncol 17(3):140-146

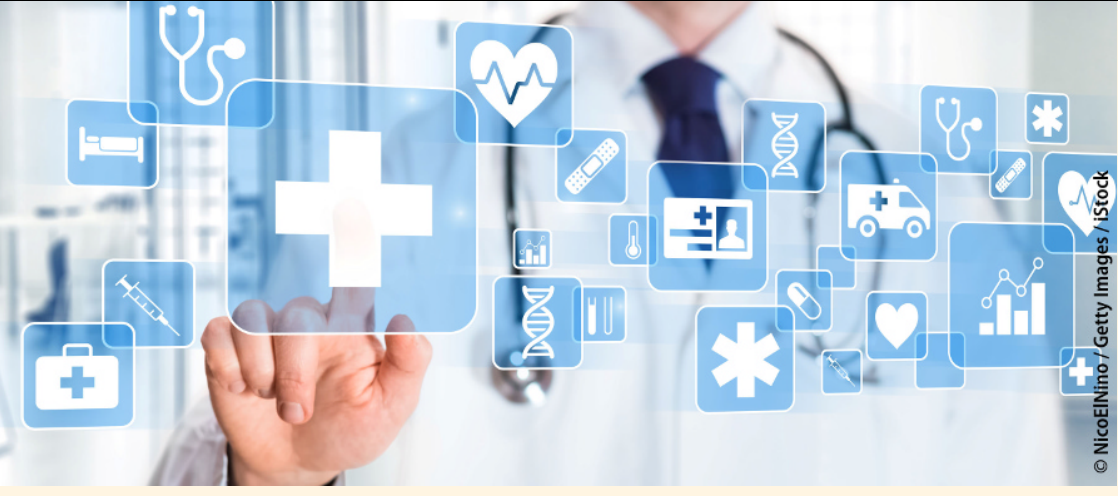

\section{DGIV-Bundeskongress, 29. Oktober 2020}

Überwindung der Sektorengrenzen - Erkenntnisse aus der CORONA-Krise

Die Deutsche Gesellschaft für Integrierte Versorgung e.V. ist ein deutschlandweit agierender Verein mit der Zielsetzung, die Integrierte Versorgung in der medizinischen, pflegerischen und sozialen Betreuung als Regelfall durchzusetzen und die dazu erforderliche Beseitigung der noch bestehenden strukturellen Hemmnisse zu befördern. Vor diesem Hintergrund lädt die DGIV zu ihrem Bundeskongress ein, der sich dem Themenkomplex CORONA (Lessons learned/ Resilienz) widmet.

Lehren aus der CORONA-Krise für die Integrierte Versorgung

- CORONA-Krise - Katalysator zur Überwindung der Sektorengrenzen

- Integrierte Versorgung - CORONA und danach

- Ethische Erkenntnisse aus der CORONAKrise

\section{Sektorenübergreifende Versorgung}

- Bewertung der Arbeitsergebnisse der Bund-Länder-Arbeitsgruppe sektorenübergreifende Versorgung

- Zu wenig Freiheit oder zu viele GruppenEgoismen? - Woran hakt es bei der Weiterentwicklung der integrierten Selektivversorgung?

- Wieviel "ambulant" können Krankenhäuser und wieviel "stationär" können Vertragsärzte leisten?
CORONA-Erfahrungen - die medizinische Perspektive

- CORONA-Erfahrungen aus erster Hand "Hotspot" Madrid

- CORONA-Erfahrungen aus der Hauptstadt Deutschlands - am Beispiel von Vivantes

- CORONA-Erfahrungen aus der Onkologie - wie sieht die Tumormedizin der Zukunft aus?

\section{Podiumsdiskussion „Wie sieht die} Roadmap nach CORONA aus?"

\section{Veranstalter}

DGIV e.V., Wartburgstraße 11, 10823 Berlin, Tel.: 030/44727080, Fax: 030/44729746,

E-Mail:info@dgiv.org

\section{Wissenschaftliche Leitung}

Prof. Dr. Stefan G. Spitzer, Dresden

Prof. Dr. Dr. Alfred Holzgreve, Berlin

Dr. Michael Meyer, Berlin

\section{Tagungsort}

Hotel Aquino, Tagungszentrum Katholische Akademie, Hannoversche Straße 5b, 10115 Berlin-Mitte

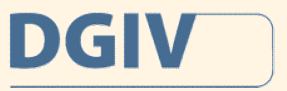

Deutsche Gesellschaft fuir Integrierte Versorgung im Gesundheitswesen e.V

Quelle und weitere Infos: www.dgiv.org 